\title{
ILCEA
}

Revue de l'Institut des langues et cultures

d'Europe, Amérique, Afrique, Asie et Australie

$16 \mid 2012$

La culture progressiste à l'époque de la guerre froide

\section{Le Chant du monde : une firme discographique au service du progressisme (1945-1980)}

Le Chant du Monde, a record company serving progressivism (1945-1980)

\section{Michèle Alten}

\section{OpenEdition}

\section{Journals}

Édition électronique

URL : http://journals.openedition.org/ilcea/1411

DOI : 10.4000/ilcea.1411

ISSN : 2101-0609

\section{Éditeur}

UGA Éditions/Université Grenoble Alpes

\section{Édition imprimée}

ISBN : 978-2-84310-232-5

ISSN : $1639-6073$

Référence électronique

Michèle Alten, « Le Chant du monde : une firme discographique au service du progressisme

(1945-1980) », ILCEA [En ligne], 16 | 2012, mis en ligne le 04 juillet 2012, consulté le 22 mars 2021.

URL : http://journals.openedition.org/ilcea/1411 ; DOI : https://doi.org/10.4000/ilcea.1411

Ce document a été généré automatiquement le 22 mars 2021.

(C) ILCEA 


\title{
Le Chant du monde : une firme discographique au service $\mathrm{du}$ progressisme (1945-1980)
}

\author{
Le Chant du Monde, a record company serving progressivism (1945-1980)
}

Michèle Alten

1 Après la seconde guerre mondiale, l'union des Alliés contre le fascisme fait rapidement long feu. Dès 1946, Churchill déclare qu'un rideau de fer vient de tomber sur l'Europe, suite à la prise de pouvoir, en Europe de l'Est, de partis communistes soutenus par Moscou (Courtois et Lazar, 1995, p. 250). Dans ce nouvel ordre du monde, les partis communistes occidentaux deviennent, après l'adoption du plan Marshall, les porteparole du camp soviétique et adoptent un discours culturel inspiré des théorisations élaborées par Moscou. Si les fondements du réalisme socialiste remontent à l'entredeux-guerres, le jdanovisme de guerre froide approfondit et élargit son message doctrinal (Aucouturier, 1998, p. 91). Le concept de progressisme, accolé à chacune des formes d'expression artistique, est officiellement lancé dans le discours politique en 1948. Comme à la fin du xix ${ }^{\mathrm{e}}$ siècle, il suppose l'affirmation d'une vision positive de l'évolution de l'humanité face à une vision de la décadence (Taguieff, 1996). Pour le camp soviétique, la décadence s'incarne dans les États-Unis et leurs alliés, alors que seuls les régimes socialistes sont porteurs d'un présent et d'un avenir meilleurs.

2 Si l'impact de cette idéologie a fait l'objet, dans le domaine des arts plastiques et de la littérature, de travaux approfondis (Baudoin et Heller, vol. 1 et 2, 1997-1998), rien de tel n'existe pour la musique. Deux ouvrages évoquent brièvement le sujet. Le premier (Caroll, 2003) propose une synthèse sur la guerre froide qui privilégie l'anticommunisme et l'existentialisme, tandis que le second (Porcile, 2001, p. 122) évoque rapidement quelques œuvres de circonstance. Pourtant les articles consacrés à la musique occupent une place importante dans les revues et hebdomadaires du PCF et le texte du Manifeste de Prague y est abondamment commenté. Soumis pour approbation aux compositeurs et critiques musicaux réunis en mai 1948, celui-ci dresse un portrait très sombre de la situation musicale dans les pays capitalistes (Alten, 2000, p. 75). En 
effet ces pays seraient atteints par une crise, liée au développement d'un secteur industriel et marchand, qui conduirait les producteurs de musique à se contenter de formules mélodiques vulgaires, corrompues et standardisées, à l'image de la musique légère américaine. À ces mélodies primaires s'opposerait une musique savante compliquée dont l'audience serait restreinte à cause de son ésotérisme et à cause du nivellement des goûts musicaux provoquée par la culture de masse. Cette situation de décadence culturelle s'expliquerait à la fois par l'abandon de valeurs identitaires nationales et par un état social défectueux. Le progressisme musical se construit donc en opposition à ce modèle décadent capitaliste. Pour faire évoluer la situation, quatre orientations sont recommandées: la mise en musique des idées progressistes, la défense du patrimoine culturel national, la priorité donnée à la musique vocale et la mise eu œuvre d'une éducation musicale des masses.

Pour appliquer ces recommandations, le PCF dispose d'un outil important, la firme d'édition musicale et discographique Le Chant du Monde. Née en 1937, puis interdite pendant la guerre, celle-ci renaît à la Libération, grâce au soutien financier du PCF. Désormais étroitement associée (jusqu'en 1982) au destin de ce parti, l'entreprise continue néanmoins à maintenir ses orientations initiales de l'avant-guerre : musiques traditionnelles du monde, jeunes compositeurs français, compositeurs soviétiques, chanson sociale et politique. Des contrats avec les compositeurs de soviétiques (édition de partitions) ainsi qu'avec la firme de disques Mélodia lui donnent un accès direct aux œuvres et aux interprètes de l'URSS (Jouvenel, 1980, p. 35). Présents dans les ventes de disques organisées par le PCF, ses disques contribuent à former la culture musicale des militants et sympathisants durant trois décennies.

Notre propos, qui se situe à la croisée de l'histoire idéologique du PCF et de l'histoire des productions phonographiques, ne prétend pas s'engager dans les problématiques relevant de l'étude de la maison d'édition, de ses budgets, de ses conflits de pouvoir, de ses arbitrages politiques et esthétiques, comme cela a été fait dans le domaine du livre (Bouju, 2010). Il ne prétend pas non plus sonder les reins et les cœurs des acheteurs de disques en étudiant la réception des œuvres. Il vise à faire sortir de l'ombre des productions musicales originales et diversifiées, proposées aux militants et sympathisants communistes comme partie intégrante d'une contre-culture engagée, dans une société qui voit l'achat d'électrophone et de microsillons se démocratiser (Tournès, 2011, p. 88). À l'instar des livres publiés aux Éditions sociales ou aux Éditions de Moscou, les disques du Chant du Monde constituent-t-ils une autorité identifiante capable de légitimer une entité collective (Roekens, 2006, p. 88) ? C'est ce que nous nous proposons d'examiner. En l'absence de dépôt légal discographique et compte tenu du caractère fragmentaire des catalogues récupérés chez le repreneur Harmonia Mundi, notre étude ne présentera aucun caractère statistique et tentera seulement de dégager les lignes de forces d'un progressisme musical, à la fois composite dans ses choix et cohérent dans son souci d'éviter l'évasion dans le divertissement et la culture standardisée.

\section{Un militantisme revendiqué}

5 Des convictions politiques affirmées peuvent choisir de s'exprimer à travers différents genres musicaux (Dalhlaus, 2004). Si la chanson révolutionnaire est le vecteur le plus 
évident de cette proclamation, elle ne saurait faire oublier certaines formes de chanson engagée ou certaines formes de musique savante au contenu très didactique.

\section{Chanter la révolution prolétarienne}

6 Célèbre par ses émissions de télévision, l'écrivain résistant non communiste Max-PolFouchet, rédige, pour le Chant du Monde, la présentation d'un disque de chants révolutionnaires du monde interprétés en français par le Groupe 17 (Groupe 17, 1967). Son texte est animé d'un souffle messianique. Il salue les quinze chants de justice, de liberté et de fraternité qui chantent l'honneur des hommes, dans une continuité de valeurs entre passé, présent et avenir. Il rappelle qu'au temps de la patrie occupée, aucune antinomie n'existait entre révolution prolétarienne et mobilisation nationale. La reproduction intégrale du texte des chants traduit une claire volonté didactique. Le répertoire choisi se caractérise par la volonté d'installer une filiation temporelle et spatiale entre les situations révolutionnaires. Le disque évoque la généalogie des prises de conscience ouvrières. En France, se sont « l'Internationale » et le "Chant des jeunes gardes » qui expriment la dénonciation de l'exploitation capitaliste et annoncent sa destruction. Après l'échec de 1905, la Révolution d'Octobre est largement célébrée. Cette victoire une fois acquise, il reste à la propager en Europe, en s'appuyant sur deux stratégies: le front uni des travailleurs et le Komintern. Au début des années 1930, le tandem communiste allemand composé par Brecht et Eisler se mobilise sur ces deux orientations (Huynh, 1998, p. 372). Mais l'avènement des fascismes transforme la situation et entraîne les emprisonnements dans les camps et la fin de la république espagnole. Cependant, même dans la détresse du camp, dans l'ignominie de la trahison ou dans l'oppression mussolinienne, l'espoir d'un futur meilleur reste présent. L'efficacité des messages passe par des musiques aux accents martiaux. Les mélodies, faciles à mémoriser, sont interprétées par un chœur homophone, avec une ponctuation rythmique et un soutien mélodique comme accompagnement. La visée est donc celle de la reproduction par des choristes amateurs militants. Les chorales de gauche, issues du Front populaire ou de la Libération, s'emparent volontiers de ce répertoire qui leur assure un succès d'audience (Jaquet, 2007, p. 11). Album emblématique de l'engagement révolutionnaire, ce disque, destiné à galvaniser les énergies, peut servir d'auxiliaire direct à l'action politique, sorte de grammaire pour l'action collective (Daré, 1996, p. 164).

\section{Le phénomène Colette Magny}

Lorsque paraît le disque connu sous le nom de sa première chanson, « Vietnam 67 », la critique unanime, du Figaro à l'Humanité et au magazine Diapason, salue l'originalité créatrice de l'auteur-compositeur-interprète Colette Magny. Trois qualités majeures lui sont reconnues : l'inventivité de ses textes, souvent composés de collages et de citations littéraires ou philosophiques, l'audace de ses musiques, inspirées du free-jazz, et enfin la qualité de sa voix, comparable à celle des chanteuses de blues noires américaines. Là s'arrête le consensus. En effet la force contestataire du disque ne permet aucun accommodement idéologique (Magny, 1967). Les messages sont virulents et mêlent dénonciation et confiance dans la révolution. Chaque chanson possède une spécificité d'écriture textuelle et musicale qui constitue la richesse de l'album. Et à l'intérieur même de chaque chanson, plusieurs styles se marient. Le parlé-chanté, utilisé pour la 
proclamation des messages didactiques, se mêle souvent à des musiques aux allures de ritournelle, utilisées pour le développement argumentaire. Lorsqu'elle défend le combat des ouvriers de Saint-Nazaire, Colette Magny joue sur deux registres contrastés : l'appel à la mobilisation s'exprime par un cri tandis qu'un rythme de valse évoque les marées noires bretonnes et la vie des ouvriers du port. Le titre «Choisis ton opium » apparaît comme un clin d'œil à la musique de gospel. Le refrain, à la pulsation très marquée, contraste avec les couplets, faits d'un jeu de citations, exprimant la supériorité intellectuelle du marxisme. La chanson de dénonciation la plus noire met en scène la détresse d'une jeunesse confrontée à un monde inhumain et elle se termine par l'espoir incarné par la révolution cubaine. L'héroïsme des communistes vietnamiens est mis en scène dans une écriture musicale tendue qui culmine dans un salut au peuple combattant. Quant aux couches moyennes des pays industrialisés, elles sont invitées à rejoindre leurs alliés naturels, les exploités. Ici encore un contraste oppose l'adresse directe, martelée à l'interlocuteur et l'évocation de la prise de conscience sur une musique dansante aux effets vocalisants.

Par son mélange d'engagement idéologique et d'inventivité poétique et musicale, cet album est l'expression d'une contre-culture engagée et progressiste. Isolée sur la scène française, Colette Magny, qui enregistre sous le label Chant du Monde jusqu'en 1984, trouvera un lointain prolongement dans le travail de l'italienne Giovanna Marini. Publié également par le chant $\mathrm{du}$ Monde, son groupe de femmes mêle radicalité politique et recherches vocales contemporaines (Marini, 1980). Dans sa Cantate de tous les jours, elle commente avec acidité la répression des manifestations, les migrations économiques et le fossé entre les exploités et les intellectuels. Mais à la fin des années 1970 la confiance dans l'avenir faiblit et le messianisme politique a vécu.

\section{Le Chant des forêts, emblème d'une nouvelle musique savante}

9 À partir du milieu des années 1950, les compositeurs les plus célèbres d'URSS, Prokofiev, Chostakovitch, Katchaturian et Kabaleski, bénéficient, en France, d'une diffusion discographique privilégiée. Créées par des interprètes prestigieux, leurs œuvres acquièrent une audience internationale grâce aux disques et aux récitals. Avant la mort de Staline en 1953, la pratique compositionnelle reste étroitement encadrée par le jdanovisme qui traque la novation «formaliste » dans l'écriture (Jdanov, 1950, p. 75). Les œuvres édifiantes composées alors laissent peu de place à l'originalité créatrice. L'une d'entre elles (Cantate de la paix, Prokofiev) est même refusée, pour médiocrité, à la gravure discographique par le directeur du Chant du monde (Jouvenel, 1980, p. 36). La recherche de canons esthétiques nouveaux, imposés par la définition d'une musique progressiste, le conduit cependant à valoriser une œuvre de commande écrite par Chostakovitch, Le Chant des forêts. Celle-ci devient le premier microsillon 33 tours pressé par la firme. Saluée dans la presse communiste comme une œuvre majeure (Jouvenel, 1951, p. 99), cette cantate ne suscite guère d'enthousiasme chez les critiques musicaux, tel Claude Rostand, qui n'y voit qu'un plat devoir d'harmonie sur des thèmes populaires (Rostand, 1951). Quant au livret, il déroule les grands thèmes de l'héroïsme soviétique et du culte de la personnalité (Prokofiev-Schostakovitch, rééd. 1998). Après 1953, lorsque les compositeurs soviétiques prennent la parole pour réclamer une autonomie créatrice, la version jdanovienne de la musique progressiste se trouve désavouée et ses défenseurs en Europe de l'Ouest disparaissent. Mais les œuvres majeures des grands compositeurs russes et soviétiques continuent, elles, à être fortement présentes dans 
les ventes militantes et à nourrir, à coté de celles des compositeurs français, la culture musicale classique du camp progressiste.

Reposant sur le double socle du marxisme et de la défense de l'URSS, le progressisme militant utilise la musique pour exalter la révolution et le modèle soviétique. Mais après l'abandon du dogmatisme musical, à partir de 1954, l'espoir dans le changement révolutionnaire de la société peut aussi inspirer des créations originales transgressives, qui s'affranchissent des langages établis pour parler d'avenir dans des formes aux audaces inconnues jusque-là dans les musiques populaires, l'engagement politique s'accompagnant alors d'un questionnement esthétique (Cecchetto, 2008, p. 17).

\section{La valorisation de l'expression musicale des peuples}

11 La solidarité internationale passe par une ouverture culturelle aux musiques du monde encore peu répandue dans l'ensemble de la société française de l'après-guerre. Les patrimoines russes et soviétiques, mais aussi américains du Nord et du Sud (voire africains ou tziganes) sont à l'honneur dans la discographie du Chant du monde. La visée n'est pas ethnomusicologique, mais il s'agit le plus souvent d'un patrimoine revisité, réactualisé, qui manifeste le lien culturel étroit qui relie les générations d'exploités.

\section{Les chœurs de l'armée soviétique}

12 En 1967, Mélodia et le Chant du Monde s'associent pour produire le disque du $50^{\mathrm{e}}$ anniversaire de la Révolution d'octobre. Le répertoire choisi se caractérise par son éclectisme: les standards folkloriques voisinent avec les chants de métier et les marches militaires (Les grands succès des choeurs de l'armée soviétique, 1967). La présentation de l'album salue le caractère exceptionnel d'une formation chorale composée de soldats qui ont payé leur tribut à la guerre. Le groupe se produit régulièrement hors d'URSS et constitue une vitrine de la suprématie socialiste. Les techniques musicales employées par les chœurs de l'armée rouge sont à la fois simples et efficaces. Lorsqu'il s'agit de chants de marche, le chœur chante à l'unisson, avec un accompagnement orchestral appuyé renforçant la dimension rythmique. Un effet de contraste est obtenu par des changements d'intensité entre les couplets. Certains sont interprétés piano alors que ceux qui suivent produisent un déferlement sonore. C'est la puissance collective maîtrisée de l'Union soviétique qui se voit ainsi affirmée. À l'inverse, lorsqu'il s'agit de chants traditionnels, l'interprétation est confiée à un chanteur soliste, le chœur se contentant alors du refrain. Dans la très célèbre "Kalinka », le ténor réussit une performance spectaculaire. Grâce à sa puissance vocale et à son souffle, il tient les notes dans la durée, en ménageant des nuances dans le phrasé. Cantonné à un rôle secondaire, le chœur joue, comme dans d'autres chants traditionnels, sur des effets d'accélération rythmique dans le refrain. Parfois le dialogue chœur-soliste est plus équilibré, comme dans « Les bateliers de la Volga » où le chœur prend le relais de la basse dans les couplets pour donner au chant une amplitude croissante. Destiné à un public français large, ce disque conjugue célébration du patrimoine et rappel de la révolution de 1917. Il représente une lecture réactualisée d'un passé national stylisé et sublimé, qui assimile Russie et URSS dans une continuité 
mythique qui élude la spécificité des expressions régionales, conformément à la volonté exprimée en 1960 par Krouchtchev (Lemaire, 2005, p. 377).

\section{La richesse du patrimoine nord-américain}

13 Si les États-Unis incarnent l'adversaire, la fascination pour leur musique s'exerce néanmoins sur des auditeurs français familiarisés avec le Jazz depuis les années 1930 (Régnier, 2006, p. 245-343). Le catalogue discographique Folkways, qui affiche plus de trente albums, présente une majorité d'albums consacrés à des musiciens noirs mais n'oublie pas cependant le folklore blanc des pionniers, des trappeurs et des marins (Folksongselection, 1964). L'interprète le plus connu de ce dernier est sans conteste Pete Seeger, membre du parti communiste américain condamné à dix ans de prison sous le maccarthisme, qui restitue, dans six enregistrements, la fraîcheur des chansons des européens expatriés. Accompagnés au banjo, à la guitare ou au violon, certains airs sont devenus des standards popularisés par les westerns. Sur des rythmes enlevés, ils symbolisent l'épopée de la conquête de l'Ouest. Mais la musique country ne se limite pas à un seul genre et c'est un répertoire varié, qui va de la ballade à la performance rythmique, qui est interprété par différents groupes instrumentaux et vocaux. L'ensemble se caractérise par une énergie vitale communicative. Sous la rubrique Rythm and Blues, le Chant du Monde propose toute une palette de chanteurs et instrumentistes noirs américains. On trouve de grands interprètes de blues comme Lightin Hopkins, Big Billy Broonzy, Champion jack Dupree, la chanteuse Ella Jenkins ainsi que le pianiste Memphis Slim. Encore peu connus en France, ces artistes, à travers leurs inventions mélodiques et rythmiques, témoignent d'une créativité musicale authentiquement populaire, dont la presse communiste rappelle qu'elle est l'expression de la souffrance et de l'oppression (Cunard, 1946). Émanation vivante de la communauté noire américaine, le jazz apparaît comme le seul cas, exemplaire, de musique du peuple pour le peuple qui ait conquis le monde. L'inscription de cette musique dans la logique du système social américain (Smadja, 2004, p. 188) semble alors secondaire.

\section{Les héritiers modernes de la tradition folklorique}

14 En Amérique du Nord et du Sud, certains auteurs-compositeurs-interprètes revendiquent une filiation avec un passé réactualisé. Des albums comportant la traduction de tous les textes sont alors proposés par le Chant du Monde. Aux États-Unis la ballade se transforme en chanson sociale sous la plume de Woodie Guthrie, chanteur qui a été, en 1940, à l'origine, avec Pete Seeger, d'un groupe de Protestsong, les Almanac Singers. De condition très modeste, il connait la vie rude des travailleurs itinérants qui sillonnent le pays en train en quête d'embauche. Il chante l'errance, la délinquance qui guette, la séparation imposée du couple, la trahison de l'ouvrier jaune qui s'oppose aux syndicats. Loin de toute idéalisation poétique, il exprime la souffrance des pauvres, à qui tout bonheur privé est interdit (Guthrie, 1972). Sa voix rauque et éraillée incarne le cri de survie des laissés pour compte de la société américaine. Aucun espoir messianique à l'horizon. Le jeu et le crime mènent le « poor boy » en prison et l'arrachement forcé au pays natal ne génère que souffrance. Le seul espoir pour les plus faibles, c'est l'organisation, par les syndicats, d'une solidarité entre les démunis, placée sous la protection de Dieu. La musique s'inspire du double héritage des colons blancs et 
des esclaves noirs. Les chansons, régulièrement rythmées évoquent la musique de danse et le chant des pionniers ou parfois le blues, longue plainte ponctuée à la guitare. Les airs, de facture simple, revendiquent une appartenance au patrimoine musical populaire, avec une dimension de dénonciation sociale nouvelle, qui influence les chanteurs de protest-song comme Bob Dylan (Dutertre, 1996, p. 67).

Une démarche assez comparable se manifeste en Amérique latine, où le poète indien Atahulpa Yupanqui chante les difficultés du petit paysan argentin. À la croisée des trois traditions culturelles indienne, espagnole et noire, il intègre ce triple héritage dans ses chansons. Accompagné à la guitare, il revisite la berceuse noire des Caraïbes ou le chant traditionnel indien, mais compose surtout des complaintes ou des milonga, dans une volonté de syncrétisme assumée. Sa virtuosité instrumentale à la guitare lui permet de rendre hommage aux créateurs du folklore rural argentin et les laissés pour compte de la richesse latifundiaire trouvent en lui un porte-parole engagé. Loin de la musique des bas-fonds urbains de la capitale, il revendique l'indianité dans son rapport à la terremère, la Pachamama (Atahualpa Yupanqui, 1969). Ouvert aux musiques du monde grâce au disque, le militant ou le sympathisant communiste peut désormais découvrir des traditions musicales originales ou revisitées qui élargissent son horizon culturel audelà de ce que lui proposent les media grand public de l'époque. La solidarité entre les peuples passe ici par une connaissance de la culture de l'autre.

\section{Une poésie française témoin de la société}

16 Pour résister à la culture de masse, aux effets jugés délétères, deux solutions peuvent être mises en œuvre : faire revivre le patrimoine national et encourager la création de qualité. Le Chant du Monde s'engage simultanément dans les deux directions.

\section{L'histoire de France par les chansons}

Dans un coffret de quatre disques, la firme propose une rétrospective de l'histoire de France, du Moyen-Âge à 1918. Deux chercheurs, France Vernillat et Pierre Barbier, ont exhumé et sélectionné les chants, présentés dans un livret détaillé. L'organisation de l'ensemble respecte la chronologie : le premier album va des Croisades à Louis XV, le second de Louis XVI à Napoléon, le troisième de la restauration à la guerre de 1870, et le dernier de la Commune à 1918 (Histoire de France par les chansons, 1982). Le déséquilibre entre les périodes s'explique par deux facteurs: la faiblesse des sources pour les périodes anciennes, mais aussi une volonté d'éclairer le présent à l'aide du passé proche. La sélection privilégie les grands événements politiques, croisades, guerres de religion, avènements de roi, guerres. La Révolution de 1789 occupe une place majeure dans le corpus, avec «le ça ira ", " la Carmagnole », " la Marseillaise », sans oublier la complainte royaliste de Louis XVI aux Français ou les chansons du Directoire. Les guerres impériales, la nostalgie de l'Empire, l'échec de 1848, la défaite de 1870 et le fonctionnement de la III ${ }^{e}$ République structurent l'évocation du XIX ${ }^{e}$ siècle. Mais ce souci chronologique n'exclut pas une analyse critique de la société. Presque inexistante jusqu'à la veille de la Révolution, celle-ci prend une place croissante dans les trois derniers disques. Après avoir stigmatisé le poids des impôts et les privilèges de l'aristocratie, les chants célèbrent les aspirations sociales des ouvriers et la répression suscitée par leurs manifestations. Toutefois le messianisme révolutionnaire est stoppé 
par la guerre de 1914. L'Union sacrée dans le patriotisme relègue à plus tard la lutte des classes et le dernier disque se termine par "la Madelon de la victoire ». La visée historique et didactique prime donc ici sur la volonté militante. L'objectif du coffret est de construire, à travers une compilation patrimoniale diversifiée, une histoire de France considérée comme partie intégrante de la culture du peuple français, car ce peuple incarne l'essence de la nation (L'année 1947, 2000, p. 412). L'interprétation des textes reste classique, avec un souci de diction et de musicalité qui témoigne d'une maitrise technique du chant. Les formes de vocalité et d'accompagnement instrumental se veulent fidèles à l'univers sonore de l'époque évoquée. Le luth ou le clavecin viennent soutenir certains airs anciens. Pour ceux qui souhaiteraient approfondir le sujet, un ouvrage de complément paraît aux éditions Max Fourny (Histoire de France par les chansons, 1982). Prémisse d'une histoire culturelle par la chanson, cette entreprise intellectuelle ambitieuse est fondée sur le choix d'objets musicaux à la fois matériels et signifiants, dont la signification se donne à lire dans une trajectoire temporelle (Pomian, 1997, p. 98).

\section{À la recherche d'une nouvelle variété française}

Dans l'après-guerre, de nouvelles formes d'expression apparaissent dans la chanson. Le Paris existentialiste de Saint-Germain-des-Prés attire les poètes, compositeurs et interprètes non-conformistes de l'époque. Le monégasque Léo Ferré trouve dans le Chant du Monde son premier éditeur discographique (Ferré, 1953). Héritières de la mélodie française, ses chansons proposent un dialogue entre voix et piano. La partie de piano se caractérise par une grande richesse musicale. Quatre thématiques principales inspirent l'auteur: Paris, la guerre d'Espagne, la bohème des poètes et la culture des humbles. Sa distance critique vis-à-vis de la société s'exprime par des évocations poétiques éloignées de tout message didactique. Ses musiques puisent à diverses sources : musique de fête populaire, de valse musette mais aussi musique savante de la première moitié $\mathrm{du} \mathrm{xx}^{\mathrm{e}}$ siècle, témoignant ainsi de la maîtrise compositionnelle du chanteur, loin des rythmes à la mode des caves parisiennes. La chanson la plus forte de l'album s'intitule "Flamenco de Paris ». D'un déchaînement pianistique qui évoque Manuel de Falla, émerge un texte âpre affirmant la solidarité avec l'ami républicain espagnol et l'espoir de la fin prochaine du franquisme. Sur un mode poétique plus distancié, on trouve une charge acerbe contre le pape Pie XII, incarnation d'une église restée silencieuse durant la seconde guerre et loin de la dureté de la vie des humbles travailleurs de banlieue. À l'opposé de l'immédiateté des chansons de combat, les textes et les musiques de Ferré font appel, pour être compris à des référents culturels dont ne disposent pas l'ensemble des militants.

En quête de nouveaux talents, le Chant du Monde fait enregistrer d'autres débutants, tel, en 1951, le chanteur interprète Mouloudji, ancien membre de l'organisation de jeunesse communiste les Faucons rouges. Mais celui-ci quitte rapidement la firme pour suivre, en 1953, Jacques Canetty. Quant à Yves Montand et Jean Ferrat, symboles des chanteurs populaires engagés à l'extrême-gauche, ils ne seront jamais édités au Chant du Monde. L'essor sans précédent du microsillon dans les années 1960 donne en effet aux chanteurs un large éventail dans le choix d'une maison de disques. Dans les années 1970, le paysage discographique issu de l'après-guerre se transforme. De nouveaux petits labels prennent place dans le champ de la dénonciation sociale par la chanson. 
Citons seulement pour mémoire l'œuvre de François Béranger, dont les textes sur la guerre d'Algérie ou l'exploitation des immigrés renouvellent le regard critique posé sur la société, à travers des thématiques nouvelles exprimées sur un mode intimiste (Béranger, 1974, 1979).

\section{La voix des poètes par la parole et par le chant}

Plusieurs formes de discographie poétique coexistent au Chant du Monde. Pour rendre hommage à la Résistance, des comédiens de théâtre, parmi les plus illustres, portent les textes des poètes résistants publiés en juin 1943 aux Éditions de Minuit (Bouju, 2010, p.181). Vilar, Negroni, Terzieff récitent Aragon, Éluard, Desnos. Une discrète ponctuation de piano accompagne cet album aux accents sombres, dramatiques et violents. Publié en 1965, il est présenté comme le symbole de l'honneur de la nation face à l'envahisseur. Dénonçant l'oppression, il célèbre le courage de ceux qui ont combattu pour la France et pour la liberté. Le régime de Vichy n'est évoqué que dans un seul poème, la collaboration étant une réalité inexprimable pour un poète patriote. Devoir de mémoire, cet hommage évacue donc, par son contenu, la complexité douloureuse de la période et vise à consolider le mythe d'une France toute entière derrière le parti des fusillés (L'honneur des poètes, 1965).

Plus intemporelle, la poésie chantée par Monique Morelli couvre huit albums au Chant du Monde, entre 1961 et 1978. Cette chanteuse-diseuse puise ses textes chez Villon, Ronsard, Bruant, Aragon, Francis Carco, Mac Orlan, Gaston Couté et Jean Clamecy. Une voix grave, à la diction impeccable et aux accents un peu canaille met en scène tout un monde de mauvais garçons et de filles de petite vertu. Loin de la vision édifiante qui fait des pauvres des êtres moraux, courageux et conscients de leur exploitation, les poètes naturalistes sont fascinés par les marginaux qui assument leur mauvaise vie, en particulier les souteneurs et les prostituées. Dans son recueil intitulé Les soliloques $d u$ pauvre, Jean-Rictus évoque la misère d'une vieille prostituée un soir de Noël. Dans cette "Prière de la Charlotte », Monique Morelli s'adresse, dans une invocation parlée de dix minutes, à la Vierge Marie. Une langue aux tournures populaires décrit la marginalité, la misère et l'aspiration à la mort. Dans sa déchéance, la prostituée évoque une pureté enfantine disparue dans les aléas de la vie. Cette veine poétique, qui exprime, sur un registre naturaliste, une marginalité assumée, bien loin de la condition ouvrière moderne, garde, par sa spécificité, une audience limitée à la partie la plus intellectuelle de la nébuleuse progressiste (Morelli, rééd. 2011).

À l'issue de ce panorama, sommes-nous en mesure d'apporter une réponse à la question de la cohérence d'une contre-culture musicale progressiste? Si certains musiciens communistes soutiennent publiquement Pierre Boulez, la démarche adornienne, inspirée du marxisme, qui fait de la musique savante radicale le révélateur du chaos social (Adorno, 1962 , p. 140) reste étrangère au discours officiel du PCF. La fidélité à la révolution prolétarienne et à sa patrie l'URSS ne supporte aucun doute, mais elle ne définit pas à elle seule la culture musicale du militant ou du sympathisant communiste. La fraternité des peuples se manifeste par l'ouverture aux musiques du monde et conduit à un élargissement culturel qui s'affranchit des limites de la culture de masse. Pour ce qui concerne la chanson française, le refus de la variété commerciale est clair. La chanson est au service d'un message, mais aussi de la qualité d'un texte et d'une musique. Patrimoniale, mémorielle, poétique ou engagée, la chanson contribue à la 
réflexion, à la prise de conscience et à l'éducation esthétique. Refusant d'envisager des publics cibles en fonction de l'âge ou du niveau culturel de l'auditeur, le Chant du Monde propose l'engagement mais aussi l'ouverture et l'exigence à tous. Malgré ses limites, ce volontarisme culturel qui incarne une alternative à la romance sucrée, au rock'n'roll et au yé-yé (Bantigny et Jablonka, 2009) exprime l'idéal à la fois mobilisateur et humaniste du progressisme musical à la française.

\section{BIBLIOGRAPHIE}

ADORNo T. W., Philosophie de la nouvelle musique, Paris, Gallimard, 1962, 220 p.

ALTEN M., Musiciens français dans la guerre froide. L'indépendance artistique face au politique, Paris, L'Harmattan, 2000, 220 p.

Aucouturier M., Le Réalisme socialiste, Paris, PUF, coll. « Que-sais-je ? », n 3320, 1998, 128 p.

BANTIGNY L. et JABLONKA I. (dir.), Jeunesse oblige. Histoire des jeunes en France, XIX ${ }^{e}-\mathrm{XX}$ siècles, Paris, PUF, 2009, $304 \mathrm{p}$.

BAUDoin A. et HELleR L., Le Réalisme socialiste de la période jdanovienne, 1947-1953, vol. 1, Les arts plastiques et leurs institutions, vol. 2, Usage à l'intérieur, images à exporter, institution littéraire, Bern, Peter Lang, 1997-1998, 418 et 394 p.

Berstein S. et Milza P. (dir.), L'année 1947, Paris, Presses de Sciences-Po, 2000, 518 p.

Bouju M. C., Lire en communiste, Les Maisons d'édition du Parti communiste français, 1920-1968, Rennes, PUR, 2010, $360 \mathrm{p}$.

Ceсchetto C., «À quoi sert une chanson si elle est désarmée ? ", Eidolon , nº 82, Bordeaux, PUR, 2008, p. 13-18.

COURTOIS S. et LAZAR M., Histoire du P.C.F, Paris, PUF, 1995, 430 p.

CUNARD N., « Musique noire », Europe, nº 4, avril 1947, p. 133-135.

DalHLaus C., "Thèses sur la musique engagée », dans Essais sur la Nouvelle musique, Genève, Éd. Contrechamps, 2004, p. 179-190.

DARÉ A., « Les partitions de l'identité », dans A. Daré (dir.), Musique et politique, les répertoires de l'identité, Rennes, PUR, 1996, p. 163-165.

DUTERTRE J. F., « Musiques traditionnelles et modernité », dans A. Daré (dir.), Musique et politique, les répertoires de l'identité, Rennes, PUR, 1996, p. 61-71.

Histoire de France par les chansons, recueillies par F. Vernillat, commentées par P. Barbier, Paris, Éd. Max Fourny, 1982, 300 p.

HuYhn P., La musique sous la République de Weimar, Paris, Fayard, 1998, 495 p.

JdAnov A., Sur la littérature, la philosophie et la musique, Paris, Éd. de la Nouvelle Critique, 1950, 98 p. JouvenEL R. DE, Souvenirs d'un sous-marin du P.C.F, Paris, Juillard, 1980, 275 p. 
-, « Le Chant des forêts de Chostakovitch et la musique soviétique », La Nouvelle Critique, $\mathrm{n}^{\circ} 24$, 1951, p. 92-99.

JAQUET F., Chanter pour dire le monde, Vénissieux, Éd. Chorale populaire de Lyon, 2007, 143 p.

LEMAIRE F. C., Le destin russe et la musique, un siècle d'histoire de la Révolution à nos jours, Paris, Fayard, 2005, $706 \mathrm{p}$.

Pomian K., « Histoire culturelle, histoire des sémiophores », dans J. P. Rioux, J. F. Sirinelli (dir.), Pour une histoire culturelle, Paris, Seuil, 1997, p. 173-100.

PORCILE F., Les conflits de la musique française (1940-1965), Paris, Fayard, 2001, 412 p.

RÉGNIER G., Jazz et société sous l'Occupation, thèse de l'université Paris 1 sous la direction de P. Ory, 3 vol., 2006, 722 p.

ROEKENS A., " Les identités collectives, l'apport des sciences sociales », dans L. Van Yperseele (dir.), Questions d'histoire contemporaine, conflits, mémoires et identités, Paris, PUF, 2006, p. 77-88.

Rostand C., dans Carrefour, no 345 du 24/04/1951.

SMADJA David, « Variations sur le jazz et la politique », Raisons politiques, Paris, Presses de Sciences Po, $\mathrm{n}^{\circ}$ 14, 2004/2, p. 179-193. Disponible sur <http://www.cairn.info/revue-raisonspolitiques-2004-2-page-179.htm>

TAGUiefF A., « Critique du progrès et pensée de la décadence. Essai de clarification des visions de l'histoire ", Mil neuf cent. Revue d'histoire intellectuelle, ${ }^{\circ}$ 14, Paris, Société des études soréliennes, 1996, p. 15-39.

TOURNÈs L., Musique, du phonographe au MP3, Paris, Éd. Autrement, réed. 2011, 188 p.

\section{Discographie}

ATAHUlPA YUPANQUI, Campesino, Duerme negrito, Le nouveau chansonnier international, le Chant du monde, GULDXS74394, 1969.

BÉRANGER François, Le monde bouge, L'escargot-ESC 323, 1974.

BÉRANGER François, Joue pas avec mes nerfs, L'escargot, ESC 390, 1979.

Chants révolutionnaires du monde par le groupe 17, Le Chant du monde, LDX-74335, 1967.

PROKOFIEV-SHOSTAKOVICH, On guard for peace, music of the totalitarian regime, CD, rééd. RCA, 09026 68772,1998

Léo Ferré chante ses premières chansons, Le Chant du monde, LDX-4351, 1953.

Folksong sélection, disque-catalogue, Folkways records, Le Chant du monde, FWX-SP-1601, 1964.

GUTHRIE Woody, Poor Boy, Folkways Records, Le Chant du monde, GU.FWX-M 50105, 1972.

Histoire de France par les chansons, France Vernillat et Pierre Barbier, Le Chant du monde, G.U. LDX74461/64, 1982.

Les grands succès des chœurs de l'armée soviétique, Mélodia, Le Chant du monde, LDX7 4353, 1967.

L'honneur des poètes, Le Chant du monde, LDX-S-6027, 1965.

MAGNY Colette, Le Chant du monde, LDX 74319, 1967.

Giovanna Marini et ses compagnes, Cantate de tous les jours, le Chant du monde, LDX 74737, 1980. 
MORELLI Monique, Chansons poétiques et réalistes, 2 CD, rééd. EPM, 2011.

\section{RÉSUMÉS}

Durant les «trente glorieuses », qui voient la France de l'après-guerre accéder à la société de consommation, le développement du disque microsillon assure à la musique une audience nouvelle. Le combat idéologique du PCF s'empare de ce vecteur culturel pour opposer une culture musicale de divertissement, d'essence capitaliste, à une musique de l'engagement et du progressisme à la française. Le contrôle qu'il exerce sur la maison de disques Le Chant du Monde lui donne les moyens d'affirmer sa différence.

L'analyse des productions concrètes de l'entreprise discographique fait apparaître une ambition culturelle qui se caractérise par une grande diversité dans les choix effectués. À côté de la chanson militante, conformiste ou d'avant-garde, sont présents des poètes français contemporains ou anciens ainsi que des musiques populaires de tous les continents. Ces trois répertoires constituent le socle du progressisme musical, élaboré au nom de la révolution, de la nation et de l'internationalisme. Une contre-culture musicale exigeante est ainsi proposée aux militants et aux sympathisants. Mais ce volontarisme culturel a des limites : il se heurte à la réalité des goûts populaires et à l'affaiblissement progressif du modèle soviétique. Il aura cependant ouvert des horizons culturels insoupçonnés à ceux qui aspiraient, au delà de leur investissement militant, à un développement personnel par l'art.

During the Thirty Glorious Years, post-war France gains access to the consumer society and, music gets a new audience through improvement of vinyl records. The ideological fight of the Communist Party makes this cultural vector its own in order to set music closely linked to political engagement and French progressivism against an entertaining musical culture representing the essence of capitalism. Consequently the control exerted on the record label Le Chant du Monde enables the communist party to assert its difference.

An analysis of production and release of this music company unveils an ambitious cultural project characterized by wide-ranging and varied choices. French contemporary or ancient poets as well as traditional music from the five continents feature with militant songs, conformist or avant-garde songs. These three different repertoires are the ground of musical progressivism, which takes root in the concept of revolution, nation and internationalism. A demanding musical counterculture is thus offered to militants and leftist sympathizers. But this cultural voluntarism is somehow limited to the actual popular tastes and to the reality of a declining soviet model. This engagement, however, opened unsuspected cultural horizons for those wanting to reach personal development through art, beyond militant involvement.

INDEX

Keywords : Chant du monde, French Communist Party, popular song, progressivism, the Cold War

Mots-clés : chanson, guerre froide, Le Chant du monde, Parti communiste français, progressisme 
AUTEUR

MICHĖLE ALTEN

Université Paris 4 - Sorbonne 